\title{
A Case of a Cardiac Resynchronization Therapy-Defibrillator Exhibiting a Lower and Alternately Variable Basic Rate
}

\author{
Keigo Iwazaki, ${ }^{1}$, Toshiya Kojima, ${ }^{2}$ MD, Takahide Murasawa, ${ }^{1}$, Jun Yokota, ${ }^{1}$, Hikaru Tanimoto, ${ }^{1}$, \\ Jun Matsuda, ${ }^{2}$ MD, Nobuaki Fukuma, ${ }^{2}$ MD, Takumi Matsubara, ${ }^{2}$ MD, Yu Shimizu, ${ }^{2}$ MD, \\ Gaku Oguri, ${ }^{2}$ MD, Eriko Hasumi, ${ }^{2}$ MD, Hitoshi Kubo, ${ }^{1}$, \\ Kyungho Chang, ${ }^{1}$ MD, Katsuhito Fujiu, ${ }^{2,3}$ MD and Issei Komuro, ${ }^{2}$ MD
}

\begin{abstract}
Summary
A cardiac resynchronization therapy defibrillator (CRT-D) (Medtronic Inc. Protecta XT) was implanted in a 67-year-old man who had cardiac sarcoidosis with extremely low cardiac function. He had ventricular tachycardia which was controlled by catheter ablation, medication and pacing. The programmed mode was DDI, lower rate was 90 beats/minute, paced AV delay was $150 \mathrm{~ms}$, and the noncompetitive atrial pacing (NCAP) function was programmed as $300 \mathrm{~ms}$.

After his admission for pneumonia and heart failure, we changed his DDI mode to a DDD mode because he had atrial tachycardia, which led to inadequate bi-ventricular pacing. After a while, there were cycle lengths which were longer than his device setting and alternately varied. We were able to avoid this phenomenon with AV delay of $120 \mathrm{~ms}$ and NCAP of $200 \mathrm{~ms}$.

NCAP is an algorithm which creates a gap above a certain period after the detection of an atrial signal during the postventricular atrial refractory period of the pacemaker. This is to prevent atrial tachycardia and repetitive non-reentrant ventriculoatrial (VA) synchrony in the presence of retrograde VA conduction. But in this case, NCAP algorithm induced much lower rate than the programmed basic lower rate. This situation produced some arrhythmias and exacerbated symptoms of heart failure. This had to be paid attention to, especially when the device was programmed at high basic heart rate.
\end{abstract}

(Int Heart J 2018; 59: 626-629)

Key words: NCAP algorithm, CRT-D

$\mathrm{C}$ ardiac implantable electronic devices use a variety of algorithms to avoid abnormal heart rhythms, but some may have unexpected consequences. Pacemaker does not exhibit a lower basic rate in a normal way. But here we describe a case of a cardiac resynchronization therapy-defibrillator (CRT-D) exhibiting a lower and alternately variable basic heart rate as a result of a particular algorithm.

\section{Case Report}

A 67-year-old man with extremely poor cardiac function (left ventricular ejection fraction 22\%) and ventricular tachycardia, was diagnosed with cardiac sarcoidosis. ${ }^{1)} \mathrm{Be}-$ fore the diagnosis, a pacemaker had been implanted for sick sinus syndrome and atrioventricular (AV) block. Catheter ablation was performed to address the ventricular tachycardia, followed by implantation of a CRT-D (Protecta XT, Medtronic Inc., Minneapolis, MN, USA: atrial lead, 6940-52 cm; right ventricular shock lead, 6945-65 $\mathrm{cm}$; and left ventricular lead, 4296-88 cm). Thereafter, his heart failure and ventricular arrhythmia were controlled with anti-arrhythmic drugs and bi-ventricular pacing.

Three years after CRT-D implantation, the patient was admitted to our hospital with pneumonia and exacerbation of heart failure. In his previous admission, he had slow ventricular tachycardia (VT) and idiopathic ventricular rhythm (IVR). So, we set his lower VT zone from 100 bpm. Furthermore, in that situation we had to overdrive VT by increasing his basic rate. ${ }^{2)}$ In the DDD mode, his lower rate was $85 \mathrm{ppm}$ as the upper limit. But we could increase his lower rate to $90 \mathrm{ppm}$ by changing his pacing mode to DDI. At the time of this admission, the CRT-D had been programmed to DDI mode with a lower rate of 90 beats/minute, $\mathrm{AV}$ delay of 150 milliseconds (ms), atrial output of $2.5 \mathrm{~V} / 0.4 \mathrm{~ms}$, atrial sensitivity of $0.3 \mathrm{mV}$, right ventricular output of $2.0 \mathrm{~V} / 0.4 \mathrm{~ms}$, right ventricular sensitivity of $0.3 \mathrm{mV}$, left ventricular output of $3.0 \mathrm{~V} / 0.4 \mathrm{~ms}$, a

From the ${ }^{1}$ Department of Medical Engineering, The University of Tokyo Hospital, Tokyo, Japan, ${ }^{2}$ Department of Cardiovascular Medicine, Graduate School of Medicine, The University of Tokyo, Tokyo, Japan and ${ }^{3}$ Department of Ubiquitous Health Informatics, Graduate School of Medicine, The University of Tokyo, Tokyo, Japan.

Address for correspondence: Toshiya Kojima, MD, Department of Cardiovascular Medicine, Graduate School of Medicine, The University of Tokyo, 7-3-1 Hongo, Bunkyo, Tokyo 113-8655, Japan. E-mail: toshiyakojima-circ@umin.ac.jp

Received for publication April 23, 2017. Revised and accepted July 5, 2017.

Released in advance online on J-STAGE April 6, 2018.

doi: 10.1536/ihj.17-235

All rights reserved by the International Heart Journal Association. 


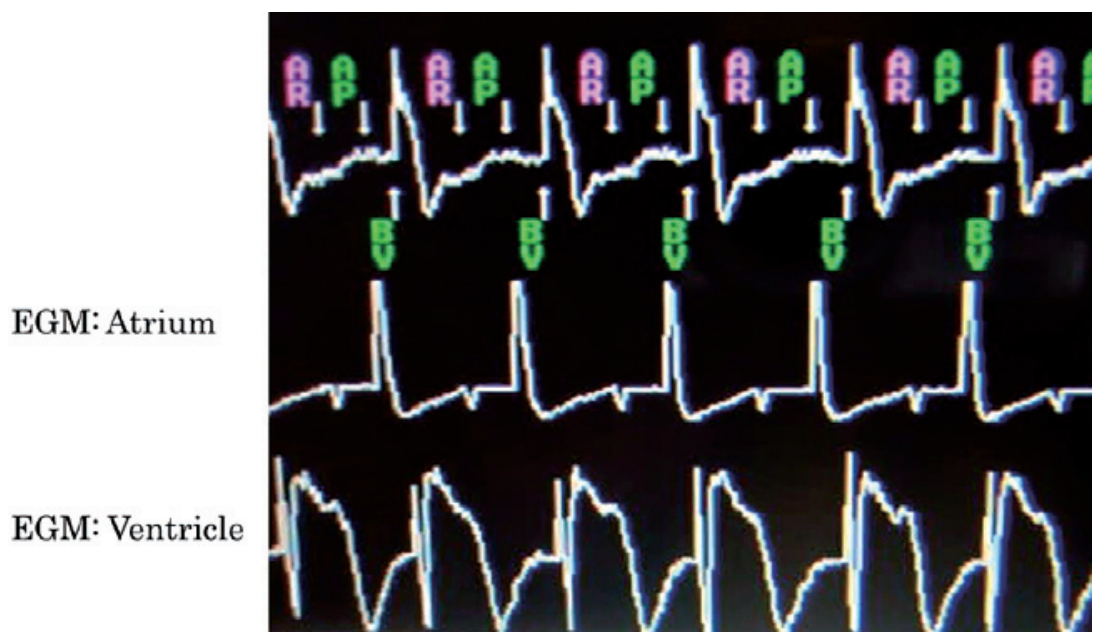

Figure 1. ECG of programmer screen. ECG exhibited a lower and alternately variable basic rate.

Table. Simulations which Induce Cycles with Longer and Alternately Variable Lengths

\begin{tabular}{ccccccccccccc}
\hline $\begin{array}{c}\text { Heart rate } \\
\text { (beats/minute) }\end{array}$ & 40 & 50 & 90 & 100 & 140 & 150 & 210 & 220 & 280 & 290 & 300 \\
\hline 80 & - & - & - & - & - & - & - & - & - & - & + \\
90 & - & - & - & - & - & - & - & + & + & + & + \\
100 & - & - & - & - & - & + & + & + & + & + & + \\
110 & - & - & - & + & + & + & + & + & + & + & + \\
120 & - & + & + & + & + & + & + & + & + & + & + \\
\hline
\end{tabular}

AV delay, $150 \mathrm{~ms}$; NCAP, 300 ms. - indicates absence of cycles with longer and alternately variable lengths; + , presence of cycles with longer and alternately variable lengths; and VP$\mathrm{AR}$, ventricular pacing-atrial refractory period. The colored box represents the baseline settings in this case.

post-ventricular atrial refractory period of $310 \mathrm{~ms}$ and non-competitive atrial pacing (NCAP) of $300 \mathrm{~ms}$. Both atrial and ventricular pacing rates were $100 \%$.

Soon after admission, the patient developed a de novo episode of atrial tachycardia (AT) with a cycle length of $550-600 \mathrm{~ms}$, which led to inadequate biventricular pacing as his CRT-D was programmed in DDI mode. In addition, his AT was being under-sensed by the atrial lead. We reprogrammed his device to DDD mode at 100 paces per minute with an atrial sensitivity of $0.15 \mathrm{mV}$ as a temporizing measure, while discussing whether catheter ablation or AV nodal ablation would be the best means of addressing his AT. This achieved satisfactory biventricular pacing, and AT was suppressed by overdrive pacing. However, the lengths of some cycles were observed to be longer than the programmed setting as well as being alternately variable, which was consistent with the findings on intracardiac electrocardiogram from the CRT-D, which also showed alternate variability in AV delay (Figure 1). In addition, his ventriculoatrial (VA) conduction became very prolonged; the ventricular pacingatrial sensing within the atrial refractory period (VP-AR) interval was $280 \mathrm{~ms}$. Nevertheless, atrial pacing fell within the NCAP interval after his basic rate was reprogrammed to 100 beats/minute, thereby delaying atrial pacing to occur just after the NCAP period. Furthermore, AV delay was automatically controlled. This case illustrates the rare development of cycles with longer and alternately variable lengths after reprogramming of a CRT-D.

We simulated several situations in order to confirm the conditions that would induce the arrhythmias observed in this case (Table). We set the AV delay to $150 \mathrm{~ms}$ and NCAP interval to $300 \mathrm{~ms}$, and held these settings constant while changing the heart rate and VP-AR interval. Under these conditions, for example, when the heart rate was over 80 beats/minute, a VP-AR interval over $210 \mathrm{~ms}$ could induce longer and alternately variable lengths. When the heart rate was over 100 beats/minute, a VP-AR interval over $150 \mathrm{~ms}$ could achieve the same outcome. These simulations suggest that VP-AR intervals that induce this phenomenon are inversely proportional to the programmed heart rate. Therefore, it is necessary to be particularly cautious if a patient's device settings are the same as these values, but the adverse effects can be avoided by setting a shorter NCAP period.

\section{Discussion}

There are many algorithms to suppress atrial arrhythmias in pacing devices. ${ }^{3)}$ Non-competitive atrial pacing involves an algorithm that creates a period during which no atrial pacing can occur after an atrial signal is detected during the post-ventricular atrial refractory period of the pacemaker (Figure 2). This is to prevent the occurrence of 

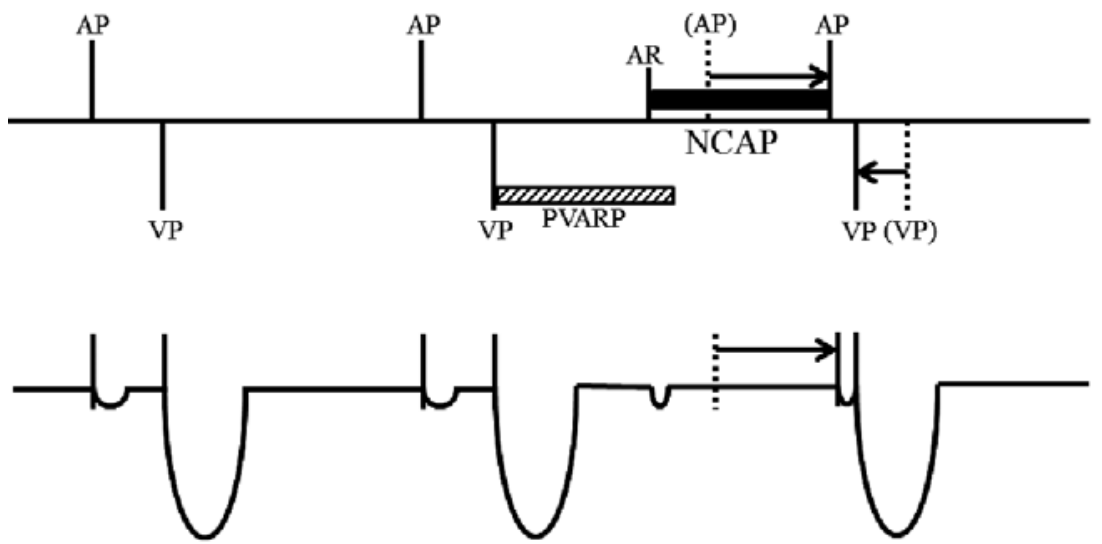

Figure 2. Non-competitive atrial pacing algorithm. Atrial pacing was expected to fall within the NCAP period as a result of the longer VP-AR interval. This led to a delay in atrial pacing and resulted in a longer cycle length than the programmed basic rate. The AV delay was automatically controlled, and cycle lengths exhibited alternate variability. AP indicates atrial pacing; VP, ventricular pacing; and $\mathrm{AR}$, atrial refractory sensing.
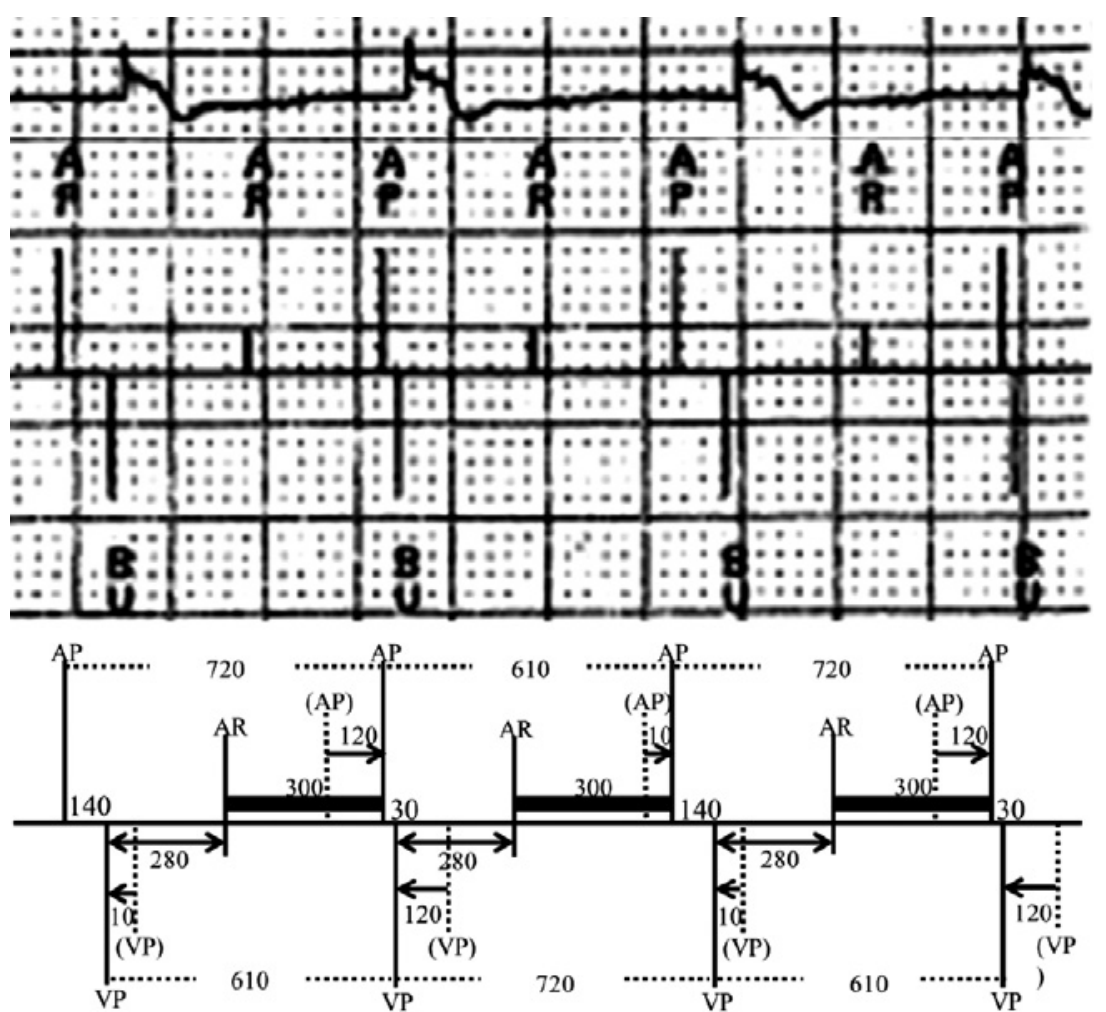

Figure 3. Intracardiac markers and interpretation. The non-competitive atrial pacing (NCAP) algorithm inhibits atrial pacing during a period after an atrial signal is detected within the post-ventricular atrial refractory period. However, in this case, NCAP delayed atrial pacing and resulted in a longer cycle length than the programmed basic rate, with the AV delay being automatically controlled. AP indicates atrial pacing; VP, ventricular pacing; PVARP, post-ventricular atrial refractory period; and AR, atrial refractory sensing.

AT and loss of atrial contraction due to repetitive nonreentrant VA synchrony in the presence of retrograde VA conduction. While the NCAP interval was empirically set to $300 \mathrm{~ms}$ in this case, there appears to be an absence of evidence to inform the choice of NCAP interval. As de- laying atrial pacing with the NCAP algorithm may also induce longer RR intervals, AV delay is automatically modulated to the shortest interval of $30 \mathrm{~ms}$ when atrial pacing is delayed (Figure 3).

Here we have reported our experience of a patient 
with an implanted CRT-D where the NCAP algorithm induced longer and alternately variable cycle lengths compared with its basic setting, ${ }^{4)}$ probably due to reprogramming to a higher basic heart rate (100 beats/minute) and a longer VP-AR interval. While this VP-AR interval might also be associated with very prolonged VA conduction and far-field $\mathrm{R}$ wave sensing of ventricular pacing or oversensing of $\mathrm{T}$ waves, atrial waves and Wenckebach phenomenon of VA conduction were nevertheless observed on this patient's surface and intracardiac electrocardiograms, respectively. We suggest that these findings were likely caused by very prolonged VA conduction due to the ventricular pacing. Whatever the cause may be, as a result of the longer VP-AR interval, atrial pacing was delayed, to occur just after the NCAP period with concurrent automatic modulation of the AV delay to the shortest $30 \mathrm{~ms}$ interval as the heart rate was being paced. As a consequence of multiple repetitions of this process, however, they induced a much slower and alternately variable rate than the programmed basic lower rate (Figure 2).

It has been reported that a heart rate lower than the basic rate might not only induce arrhythmias due to the loss of overdrive effect in atrial and ventricular tachycardia, ${ }^{5,6)}$ but may also exacerbate symptoms of heart failure due to the AV delay being too short. ${ }^{78)}$ However, we were able to avoid this phenomenon with an AV delay of 120 ms and NCAP of $200 \mathrm{~ms}$. Setting off NCAP might be another option to avoid that. As described above, NCAP is effective in preventing AT and loss of atrial contraction, but occasionally it may work to the detriment of cardiac rhythm and function. While we sometimes set higher heart rates to avoid arrhythmias and heart failure, this strategy requires particular care and attention when the NCAP algorithm is to be implemented.

\section{Disclosures}

Conflicts of interest: None.

\section{References}

1. Matsuda J, Fujiu K, Roh S, et al. Cardiac sarcoidosis diagnosed by incidental lymph node biopsy. Int Heart J 2017; 58: 140-3

2. Fujiishi T, Niwano S, Murakami M, et al. Efficacy and limitations of tachycardia detection interval guided reprogramming for reduction of inappropriate shock in implantable cardioverterdefibrillator patients. Int Heart J 2016; 57: 304-9.

3. Miki Y, Ishikawa $\mathrm{T}$, Inoue $\mathrm{N}$, et al. Efficacy of consistent atrial pacing algorithm for suppression of atrial arrhythmias in patients with sick sinus syndrome and atrial fibrillation. Int Heart J 2008; 49: 273-80.

4. Barold SS, Levine PA, Isaeff DM, Betzold R. Case report: complex arrhythmia induced by noncompetitive atrial pacing algorithm of DDDR pacemaker. J Interv Card Electrophysiol 2001; 5: 431-4.

5. Barold SS, Levine PA. Pacemaker repetitive nonreentrant ventriculoatrial synchronous rhythm. A review. J Interv Card Electrophysiol 2001; 5: 45-58.

6. Defaye P, Dournaux F, Mouton E. Prevalence of supraventricular arrhythmias from the automated analysis of data stored in the DDD pacemakers of 617 patients: the AIDA study. The AIDA Multicenter Study Group. Automatic Interpretation for Diagnosis Assistance. Pacing Clin Electrophysiol 1998; 21: 250-5.

7. Gold MR, Feliciano Z, Gottlieb SS, Fisher ML. Dual-chamber pacing with a short atrioventricular delay in congestive heart failure: a randomized study. J Am Coll Cardiol 1995; 26: 96773.

8. Ishikawa T, Sumita S, Kimura K, et al. Optimization of atrioventricular delay and follow-up in a patient with congestive heart failure and with bi-ventricular pacing. Jpn Heart J 2001; 42: 781-7. 